\title{
Circular RNA circ-CCT3 promotes hepatocellular carcinoma progression by regulating the miR-1287-5p/TEAD1/PTCH1/LOX axis
}

\author{
WENNAN LIN ${ }^{1}$, TIANYU ZHANG ${ }^{2}$, GUOXU DING $^{1}$, LIGUO HAO ${ }^{3}$, BINGQUAN ZHANG ${ }^{1}$, \\ JING YU ${ }^{4}$, YU PANG ${ }^{5}$, FENG GENG $^{6}$, LAN ZHAN ${ }^{5}$, MINGLU ZHOU ${ }^{1}$, QIYU YAN ${ }^{1}$, \\ YUGUANG WANG ${ }^{2}, \mathrm{CHUNLEI} \mathrm{ZHENG}^{7}$ and $\mathrm{HUI} \mathrm{LI}^{8}$ \\ Departments of ${ }^{1}$ General Practice and ${ }^{2}$ Computed Tomography, The Second Affiliated Hospital of \\ Qiqihar Medical University; ${ }^{3}$ Department of Molecular Imaging, Qiqihar Medical University; \\ Departments of ${ }^{4}$ Gastroenterology, ${ }^{5}$ Neurology, ${ }^{6}$ Pharmacy, ${ }^{7}$ Oncology and ${ }^{8}$ Electrophysiology, \\ The Second Affiliated Hospital of Qiqihar Medical University, Qiqihar, Heilongjiang 161000, P.R. China
}

Received September 22, 2020; Accepted March 1, 2021

DOI: $10.3892 / \mathrm{mmr} .2021 .12014$

\begin{abstract}
Hepatocellular carcinoma (HCC) is characterized by a poor prognosis because of its insensitivity to radiation and chemotherapy. Recently, circular RNAs (circRNAs) have been found to serve important roles in hepatocellular carcinogenesis. circ-CCT3, a novel circRNA, was screened from the differential tissue expression results of a circRNA microarray. Relative expression levels of circ-CCT3 in specimens and cell lines were evaluated by reverse transcription-quantitative PCR and the relationship between circ-CCT3 and prognosis was analyzed by Kaplan-Meier curves. The oncogenic role of circ-CCT3 was confirmed in HCC cells through a cell counting kit-8 (CCK-8) assay, a colony formation assay, acridine orange/ethidium bromide double fluorescence staining, flow cytometry, a wound-healing assay and a Transwell assay. Bioinformatics prediction and luciferase reporter assays validated that circ-CCT3 facilitated HCC progression through the miR-1287-5p/TEA domain transcription factor 1 (TEAD1) axis. TEAD1 could then directly activate patched 1 and lysyl oxidase transcription, as analyzed by chromatin immunoprecipitation and luciferase reporter assays. The present study identified a novel circRNA, circ-CCT3, which may be used as a potential therapeutic target for HCC.
\end{abstract}

\section{Introduction}

Hepatocellular carcinoma (HCC) is a digestive tumor with a high morbidity and mortality worldwide. HCC is the

Correspondence to: Dr Wennan Lin, Department of General Practice, The Second Affiliated Hospital of Qiqihar Medical University, 37 Zhonghuaxi Road, Qiqihar, Heilongjiang 161000, P.R. China

E-mail: wesleykm@sina.com

Key words: hepatocellular carcinoma, circ-CCT3, miR-1287-5p, TEA domain transcription factor 1 commonest cancer of the liver and the sixth commonest cancer in worldwide (1). The overall survival of HCC patients is poor due to its unresponsiveness to early diagnosis and its drug resistance (2). Over the past few decades, efforts have been made to characterize the molecular and genetic mechanisms of HCC (3). However, further research to uncover new therapeutic targets for HCC is urgently needed.

Accumulated evidence has demonstrated that a type of non-coding RNA, circular RNA (circRNA), is commonly expressed in human tissues and participates in multiple biological processes (4). Abnormal expression of circRNAs is associated with cancer pathogenesis, including HCC development and progression $(5,6)$. circRNAs have been identified to function as miRNA sponges (7-9) and circRNAs might indirectly modulate target gene expression by sponging miRNAs at the post-transcriptional level. The present study performed circRNA sequencing to reveal the dysregulated circRNAs in HCC tissues compared with nontumorous samples. circ-CCT3 originates from chr1:156303337-156304709 in the host gene CCT3. The spliced length of circ-CCT3 is $211 \mathrm{nt}$. circ-CCT3 has not been studied previously, to the best of the authors' knowledge. The present study identified that upregulation of circ-CCT3 facilitated HCC cell progression by sponging miR-1287-5p to regulate TEA domain transcription factor 1 (TEAD1) expression. TEAD1 transcriptional activity is widely believed to be modulated by the presence or absence of nuclear Yes-associated protein (YAP)/transcriptional activator with PDZ-binding domain (TAZ) (10). The present study found that TEAD1 could directly activate patched 1 (PTCH1) and lysyl oxidase (LOX) transcription. In brief a novel circRNA, circ-CCT3 was identified, which may be a potential therapeutic target for HCC.

\section{Materials and methods}

Study participants. A total of $68 \mathrm{HCC}$ tissues and neighboring nontumorous specimens ( $\geq 2 \mathrm{~cm}$ from the edge of the tumor) were collected from HCC patients who underwent partial 
hepatectomy at the Second Affiliated Hospital of Qiqihar Medical University between January 2013 and June 2015 and written informed consent was obtained from all patients. There were 57 males and 11 females in the cohort. The average age was 59.2 years (range, $35-78$ years). All patients were followed up after surgery until mortality or survival of $>5$ years. All tissues were snap-frozen and then transferred into a $-80^{\circ} \mathrm{C}$ freezer. The present study was authorized by the Institutional Review Board of the Second Affiliated Hospital of Qiqihar Medical University.

circRNA sequencing. Total RNA was extracted from four pairs of HCC tissue samples with TRIzol ${ }^{\circledR}$ (Thermo Fisher Scientific, Inc.) according to the manufacturer's protocols. RNA quantity and quality were then determined using an ND-1000 spectrophotometer (NanoDrop Technologies; Thermo Fisher Scientific, Inc.). RNA integrity number (RIN) analysis was performed using an Agilent 2100 Bioanalyser and RNA 6000 LabChip kit with Agilent 2100 Expert software (Agilent Technologies). The isolated RNA from tissue samples with RIN $\geq 7$ was considered usable in the study. A cDNA library was established using an RNA Sample Prep kit and circRNA-seq was conducted on an Illumina HiSeq 2500 platform (Illumina, Inc.). The original sequencing data were preprocessed through cutadapt v3.2 (cutadapt.readthedocs. io/en/stable/) and FastQC v0.11.9 (bioinformatics.babraham. ac.uk/projects/fastqc/) and clean readings were subsequently recorded in hg38 and circBase through TopHat 2.1.1 (ccb.jhu. edu/software/tophat/index.shtml) and MapSplice 2.2.1 (netlab. uky.edu/p/bioinfo/MapSplice2). The circRNAs with different expression levels were determined through the $\mathrm{R}$ package EdgeR v3.14.0 (R-project.org/) (11) with a fold alternation >2 and $\mathrm{P}<0.05$.

HCC cells and transfection. Normal human liver cells (Chang) and liver cancer cells (HepG2, Huh7, HCCLM3 and SK-Hep-1) were purchased from the Chinese Academy of Sciences. Cells were cultivated at $37^{\circ} \mathrm{C}, 5 \% \mathrm{CO}_{2}$ in a mixture composed of $10 \%$ fetal bovine serum (FBS) and 90\% DMEM (HyClone; Cytiva). Cells were harvested at $70-80 \%$ confluence to perform the subsequent experiments.

Short hairpin RNA (shRNA) against circ-CCT3 (sh-CCT3-1/-2), TEAD1 (sh-TEAD1), sh negative control (NC), miR-1287-5p mimics, inhibitor, mimics-NC and inhibitor-NC were synthesized by Shanghai GenePharma Co., Ltd. TEAD1 vector and its NC vector were purchased from Shanghai GeneChem Co., Ltd. Transient transfection was performed using Lipofectamine ${ }^{\circledR} 3000$ (Invitrogen; Thermo Fisher Scientific, Inc.) according to the manufacturer's protocols. The targeted sequences of shRNA-circ-CCT3 were: sh-circ-CCT3-1, 5'-AGTTTTATTAGAGACAAAGCA-3' and sh-circ-CCT3-2, 5'-TAATTATTCTTGACTATTGCA-3'.

Reverse transcription-quantitative (RT-q) PCR and western blotting. TRIzol ${ }^{\circledR}$ (Thermo Fisher Scientific, Inc.) was used to isolate total RNA from tissue samples and cells according to the manufacturer's instructions. RNA isolation was performed at $48 \mathrm{~h}$ after transfection (the cells were grown to $\sim 80 \%$ density). cDNA was synthesized by Capital-Bio with Oligo (dT) in accordance with the manufacturer's protocol. Primers were designed by Shanghai Sangong Pharmaceutical Co., Ltd. RT-qPCR experiments were conducted on a real-time system using SYBR Green Master Mix (Roche $\mathrm{GmbH}$ ) following the manufacturer's instructions. The reaction volume was $10 \mu \mathrm{l}$. Thermocycling conditions were as follows: $90^{\circ} \mathrm{C}$ for $5 \mathrm{~min}$, then $90^{\circ} \mathrm{C}$ for $15 \mathrm{sec}, 60^{\circ} \mathrm{C}$ for $30 \mathrm{sec}$ for 45 cycles. For circRNA and mRNA quantification, GAPDH was used as the internal reference. For miRNA quantification, U6 was used as the internal control. The primers used were as follows: circ-CCT3 forward, 5'-AATTAGCCGGACCCAGGATG-3' and reverse, 5'-ACAATGCCTCCCATTGGGTC-3'; CCT3 forward, 5'-AAGTCCATGATCGAAATTAGCCG-3' and reverse, 5'-TGCTCAGCTACAGACAGCATT-3'; TEAD1 forward, 5'-ATGGAAAGGATGAGTGACTCTGC-3' and reverse, 5'-TCCCACATGGTGGATAGATAGC-3'; PTCH1 forward, 5'-CCAGAAAGTATATGCACTGGCA-3' and reverse, 5'-GTGCTCGTACATTTGCTTGGG-3'; LOX forward, 5'-CGGCGGAGGAAAACTGTCT-3' and reverse, 5'-TCG GCTGGGTAAGAAATCTGA-3'; GAPDH forward, 5'-GGG AGCCAAAAGGGTCAT-3' andreverse,5'-GAGTCCTTCCAC GATACCAA-3' and U6 forward, 5'-ATTGGAACGATACA GAGAAGATT-3' and reverse, 5'-GGAACGCTTCACGAAT TTG-3'. Each reaction was performed in triplicate. The $2^{-\Delta \Delta C} \mathrm{q}$ method was employed to analyze gene expression (12).

Immunoblotting was carried out as per our previous study (13). In brief, the cells were lysed with RIPA lysis buffer (Beyotime Institute of Biotechnology). BCA method was used to detect the concentration of proteins. Protein (30 $\mu \mathrm{g} /$ lane) was fractionated by SDS-PAGE vertical electrophoresis (10\% gel), followed by transfer onto a $0.45 \mu \mathrm{m}$ PVDF membrane. The membrane was blocked with $5 \%$ skimmed milk (BD Biosciences) diluted in Tris-buffered saline containing $0.05 \%$ Tween-20 for $1 \mathrm{~h}$ at room temperature and then probed with primary antibodies to TEAD1 (1:1,000; cat. no. ab133533; Abcam) and GAPDH (1:10,000; cat. no. ab181602; Abcam) at $4^{\circ} \mathrm{C}$ overnight. After washing and incubating with horseradish peroxidase-conjugated goat anti-rabbit IgG secondary antibody (1:5,000; cat. no. ab6721; Abcam) for $2 \mathrm{~h}$ at room temperature, BeyoECL Plus kit (Beyotime Institute of Biotechnology) was used to visualize the blots. Image J 1.50i software (National Institutes of Health) was used to analyze the blots.

Dual-luciferase reporter gene assay. To explore the interaction between the circ-CCT3/TEAD1 3'-UTR and miR-1287-5p, the circ-CCT3/TEAD1 3'-UTR vector was constructed using the pmirGLO Luciferase Reporter Vector (Promega Corporation) according to the manufacturer's protocol. Transfections were performed in accordance with the instructions of Lipofectamine ${ }^{\circledR} 3000$ (Invitrogen; Thermo Fisher Scientific, Inc.) to co-transfect circ-CCT3/TEAD1 3'-UTR vector with NC miRNA or miR-1287-5p mimics into cells. The Dual-Luciferase Reporter Assay System (Promega Corporation) was used to evaluate the relative luciferase signal at $36 \mathrm{~h}$ post-transfection. The specific target activity was expressed as the relative activity ratio of firefly luciferase to Renilla luciferase. miR-1287-5p mimics sequence was 5'-UGCUGGAUCAGUGGUUCGAGUC-3'. Mimics-NC sequence was 5'-UUCUCCGAACGUGUCACGU-3' (Shanghai GenePharma Co., Ltd.). 
Chromatin immunoprecipitation (ChIP). DNA and proteins were crosslinked in cells using formaldehyde (Sinopharm Chemical Reagent Co., Ltd.). Cell lysates were sonicated to generate chromatin fragments of $200-300 \mathrm{bp}(20 \mathrm{kHz}$; 4 pulses of $12 \mathrm{sec}$ each, followed by $30 \mathrm{sec}$ rest on ice between each pulse). Centrifugation was performed at 14,000 $\mathrm{x} g$ for $10 \mathrm{~min}$ at $4^{\circ} \mathrm{C}$ before protease inhibitor mixture II buffer (EMD Millipore) at a ratio of 9:1 and $60 \mu \mathrm{l}$ protein $\mathrm{G}$ agarose was added and then cultured at $4^{\circ} \mathrm{C}$ for $1 \mathrm{~h}$. After removing the agarose by centrifugation at $4,000 \mathrm{x}$ g for $1 \mathrm{~min}$ at $4^{\circ} \mathrm{C}$, antibody against TEAD1 (1:80; cat. no. ab133533; Abcam) or IgG (1:50; cat. no. ab6721; Abcam) was added into the supernatant. The precipitated DNA was purified using the ChIP DNA Clean \& Concentrator kit (A\&D Technology) according to the manufacturer's protocol. The precipitated chromatin DNA was recovered and assessed by qPCR.

Cell counting kit-8 (CCK-8) and colony-forming assays. CCK-8 was used to measure the viability of treated cells. Cells were placed in 96-well plates and then $10 \mu \mathrm{l}$ of CCK-8 solution (Dojindo) was added to each well according to the manufacturer's instructions. The absorbance was estimated using a spectrophotometer at $450 \mathrm{~nm}$. Transfected cells were plated in $2.5 \mathrm{~cm}$ dishes for 10 days. The visible colonies were then fixed and stained for observation.

Apoptosis assays. HCCLM3 and Huh7 cells (10,000 cells/well) transfected accordingly were harvested by trypsin (HyClone; Cytiva) digestion. A total of $5 \mu \mathrm{l}$ Annexin V-FITC and $10 \mu \mathrm{l}$ PI were added to each sample for $20 \mathrm{~min}$ in the dark at room temperature. Afterwards, cell apoptosis was assessed by flow cytometry (FCM; FACScan; BD Biosciences). FlowJo v10 software (Tree Star, Inc.) was used for apoptosis analysis. The percentage of early + late apoptotic cells was deemed as apoptotic rate. For the acridine orange/ethidium bromide (AO/EB) double fluorescence staining assay, transfected cells were cultured in an incubator, followed by staining with prepared $\mathrm{AO} / \mathrm{EB}$ mixing solution (1:1) at room temperature for $5 \mathrm{~min}$ (Beijing Solarbio Science \& Technology Co., Ltd.).

Wound healing assay. For wound-healing assay, $2 \times 10^{5}$ transfected HCCLM3 and Huh7 cells were seeded into a $2.5 \mathrm{~cm}$ dish and cultured with complete medium until $90 \%$ confluency. Wounds were made using a $200 \mu \mathrm{l}$ sterile pipette tip. The detached cells were removed by washing twice with PBS and then, the cells were cultured with serum-free medium for $24 \mathrm{~h}$. A light microscope was used for visualizing and capturing images (magnification, x100). ImageJ version 1.50i software (National Institutes of Health) was used to analyze cell migration.

Transwell experiments. A Transwell chamber (Corning Inc.) was used for the migration assay (with $8-\mu \mathrm{m}$ polycarbonate nucleopore filters). For the invasion assay, the Transwell compartment was coated with Matrigel (precooled at $4^{\circ} \mathrm{C}$ overnight) and placed in an incubator at $37^{\circ} \mathrm{C}$ for $4 \mathrm{~h}$ to form a reconstructed basement membrane. The cells $\left(\sim 5 \times 10^{4}\right.$ cells $)$ resuspended in serum-free medium were seeded into the top compartment of the Transwell chamber, while the bottom compartment was filled with medium containing 10\% FBS.
After incubation at $37^{\circ} \mathrm{C}$ for $24 \mathrm{~h}$, the cells on the upper side of membrane were removed. The migrated/invaded cells were fixed with paraformaldehyde for $20 \mathrm{~min}$ at room temperature, stained with $0.5 \%$ crystal violet for $20 \mathrm{~min}$ and measured with a light microscope (Olympus Corporation).

Bioinformatics and statistical analyses. Target prediction was performed by starBase v2.0 software (starbase.sysu.edu.cn), which is based on seed region matching of miRNAs (14). The circRNA-miRNA interaction network was drawn by Circular RNA Interactome software v2020-01-30 (15). The Cancer Genome Atlas (TCGA) database (cancer.gov/about-nci/organization/ccg/research/structural-genomics/tcga/using-tcga) was analyzed by starBase v2.0 software. Kaplan-Meier estimate was used to measure the overall survival rate of patients. The differences between two groups and multiple groups were analyzed by using unpaired t-tests and one-way analysis of variance with Tukey's test, respectively. Pearson's correlation coefficient analysis was performed to analyze the expression correlation between circ-CCT3, miR-1287-5p and TEAD1. $\mathrm{P}<0.05$ was considered to indicate a statistically significant difference.

\section{Results}

circ-CCT3 is elevated in HCC tissues and indicates poor prognosis. A circRNA microarray was carried out to identify the dysregulated circRNAs in HCC tissue specimens. The heatmap for the top 20 up/downregulated circRNAs is shown in Fig. 1A. Then, RT-qPCR was performed to explore the three most elevated circRNAs (hsa_circRNA_100364, hsa_circRNA_102777 and hsa_circRNA_102723) in 20 paired HCC/nontumorous specimens. As Fig. 1B-D shows, all three circRNAs were upregulated in HCC specimens. Hsa_circRNA_100364 was the most overexpressed circRNA and was selected for subsequent study. Hsa_circRNA_100364 was spliced from exons 4-6 of the CCT3 gene. The full sequence length of hsa_circRNA_100364 (circ-CCT3) was $211 \mathrm{nt}$ (Fig. 1E). Additionally, it was found that the half-life of circ-CCT3 was longer than that of its linear isoform (CCT3 mRNA; Fig. 1F). Moreover, circ-CCT3 was more stable than CCT3 mRNA after treatment with RNase R (Fig. 1G). As shown in Fig. 1H, circ-CCT3 expression was significantly higher in HCC tissue samples than in nontumorous tissues. The 68 HCC patients were divided into two groups based on the median cutoff to analyze the clinical significance of circ-CCT3 expression in tissues. It was found that high circ-CCT3 expression was linked to a worse overall survival rate $(\mathrm{P}=0.001)$ for patients after surgical resection (Fig. 1I). circ-CCT3 expression was higher in Huh7, HCCLM3 and SK-Hep-1 cells compared with Chang cells (Fig. 1J), suggesting that circ-CCT3 might promote HCC progression. Therefore, HCCLM3 and Huh7 cells were considered suitable for use in knockdown experiments.

circ-CCT3 regulates $H C C$ cell progression. As expected, two shRNAs led to downregulation of circ-CCT3 expression in HCCLM3 and Huh7 cell lines, as confirmed by RT-qPCR (Fig. 2A). In contrast, CCT3 mRNA expression was unaffected following transfection (Fig. 2B). The functional 
A
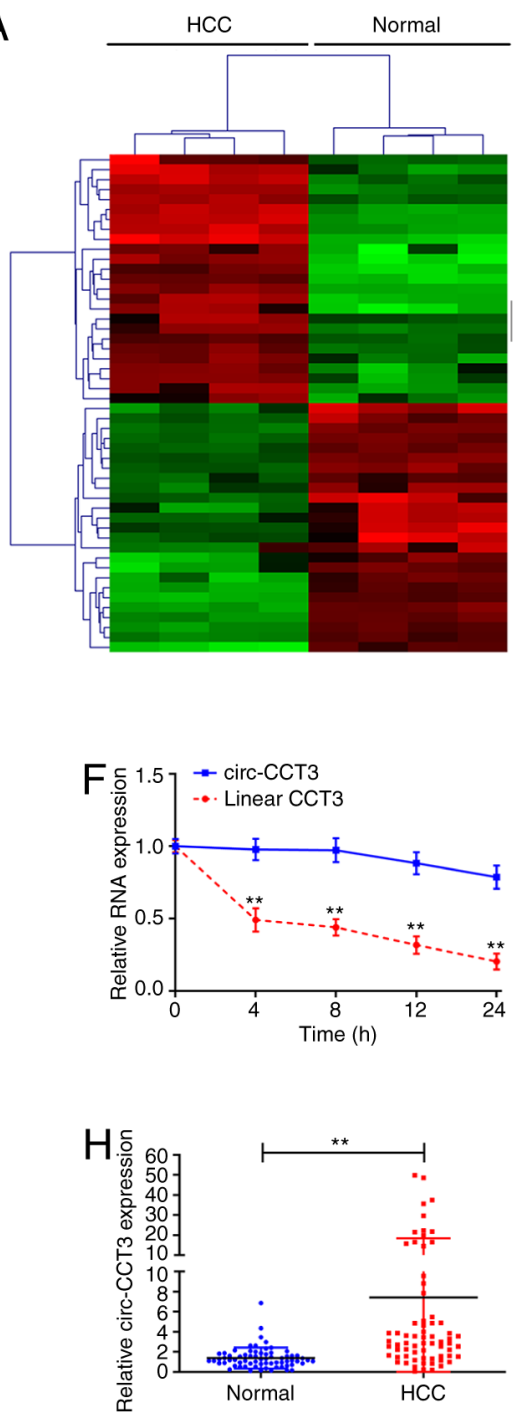

B

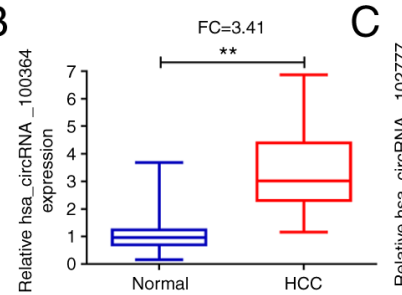

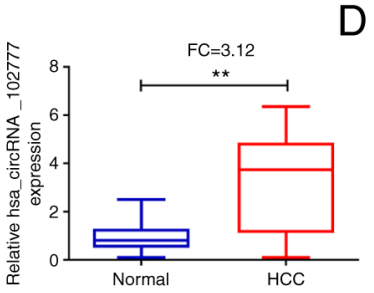

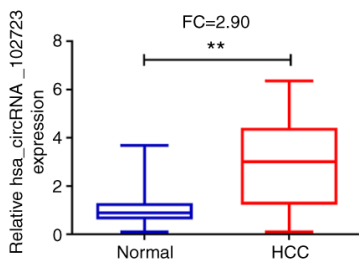

E

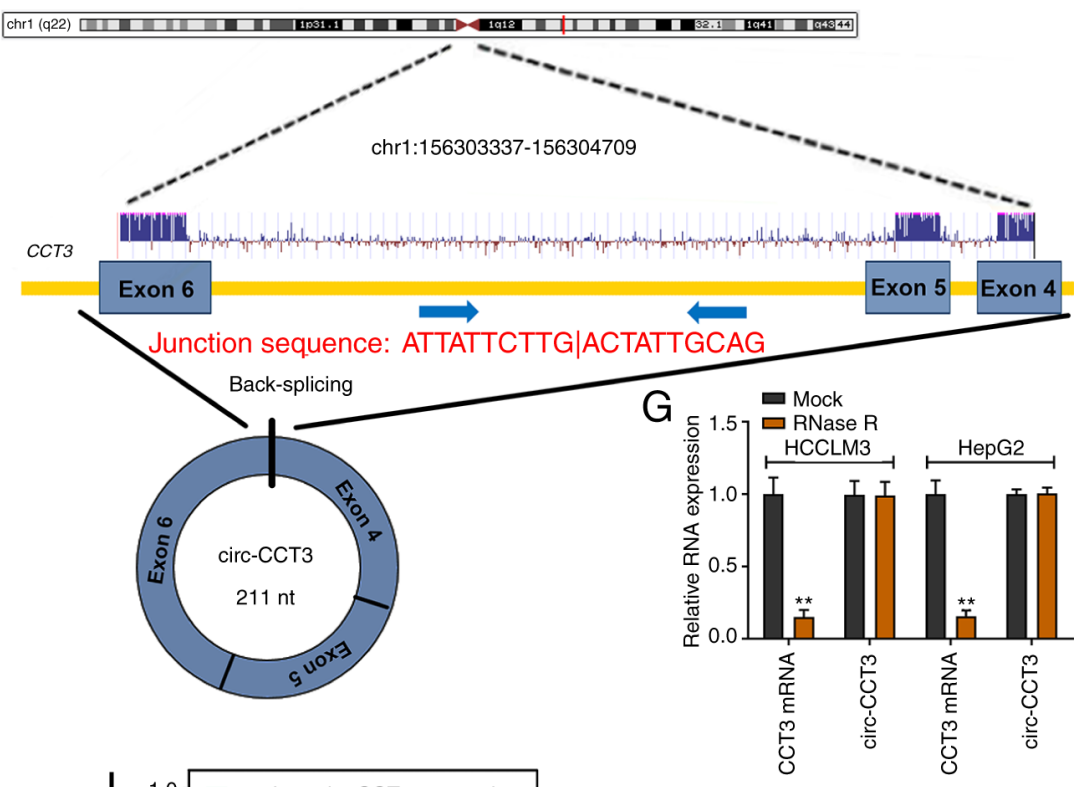

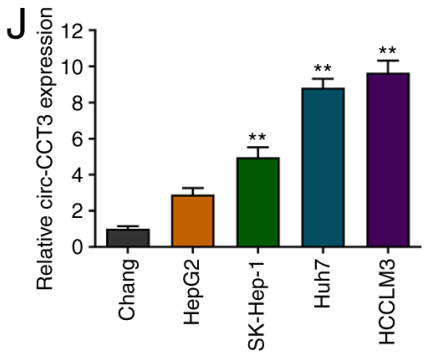

Figure 1. circ-CCT3 expression in HCC tissues and cells and its clinical importance. (A) Clustered heatmap showing HCC tissue-specific circRNAs. The expression of (B) hsa_circRNA_100364, (C) hsa_circRNA_102777 and (D) hsa_circRNA_102723 was evaluated by RT-qPCR in HCC tissues/adjacent nontumorous tissues. (E) Schematic representation of circ-CCT3 formation. (F) Relative circ-CCT3 and linear CCT3 mRNA expression at different time points. (G) circ-CCT3 was resistant to RNase R digestion in HCC cells. (H) circ-CCT3 expression in 68 pairs of HCC tissues/adjacent nontumorous tissues by RT-qPCR. ${ }^{* *} \mathrm{P}<0.01$ vs. Mock. (I) Kaplan-Meier analysis with log-rank test for overall survival in HCC patients according to circ-CCT3 expression. (J) Relative expression of circ-CCT3 in HCC and normal cells by RT-qPCR. ${ }^{* *} \mathrm{P}<0.01$ vs. Chang. circ, circular; HCC, hepatocellular carcinoma; has, human; RT-qPCR, reverse transcription-quantitative PCR; FC, fold change.

experiments were then conducted. Reduced circ-CCT3 markedly suppressed cell proliferation, as analyzed by CCK-8 and colony-forming assays (Fig. 2C and D). Consistently, AO/EB staining and flow cytometry assays showed that downregulation of circ-CCT3 led to increased apoptosis in HCCLM3 and Huh7 cells (Fig. 2E and F). Notably, HCCLM3 and Huh7 cells had decreased migratory potential following knockdown of circ-CCT3 as demonstrated by Transwell and scratched wound assays (Fig. 2G and $\mathrm{H}$ ). The Transwell assay also identified decreased invasion of cells in the circ-CCT3-KD group compared with the control group.

circ-CCT3 upregulates TEAD1 expression via sponging miR-1287-5p in HCC. circ-CCT3 regulates the growth and aggressiveness of $\mathrm{HCC}$ cells, but the underlying mechanism remains to be elucidated. As shown in Fig. 3A, circ-CCT3 was primarily localized in the cytoplasm of cells. An online database predicted the miRNAs that may be bound to circ-CCT3. The expression of predicted miRNAs was evaluated after knockdown of circ-CCT3 in HCCLM3 and Huh7 cell lines. The data indicated that only miR-1287-5p expression levels were enhanced in circ-CCT3-depleted cells (Fig. 3B). Additionally, miR-1287-5p expression was decreased in HCC tissue samples (Fig. 3C). Luciferase reporter vectors were constructed a for wild-type (wt) and mutant (mut) circ-CCT3. The vectors were cotransfected with miR-1287-5p mimics or mimics-NC in HCCLM3 and Huh7 cells. After 36 h, miR-1287-5p mimics notably inhibited luciferase activity 

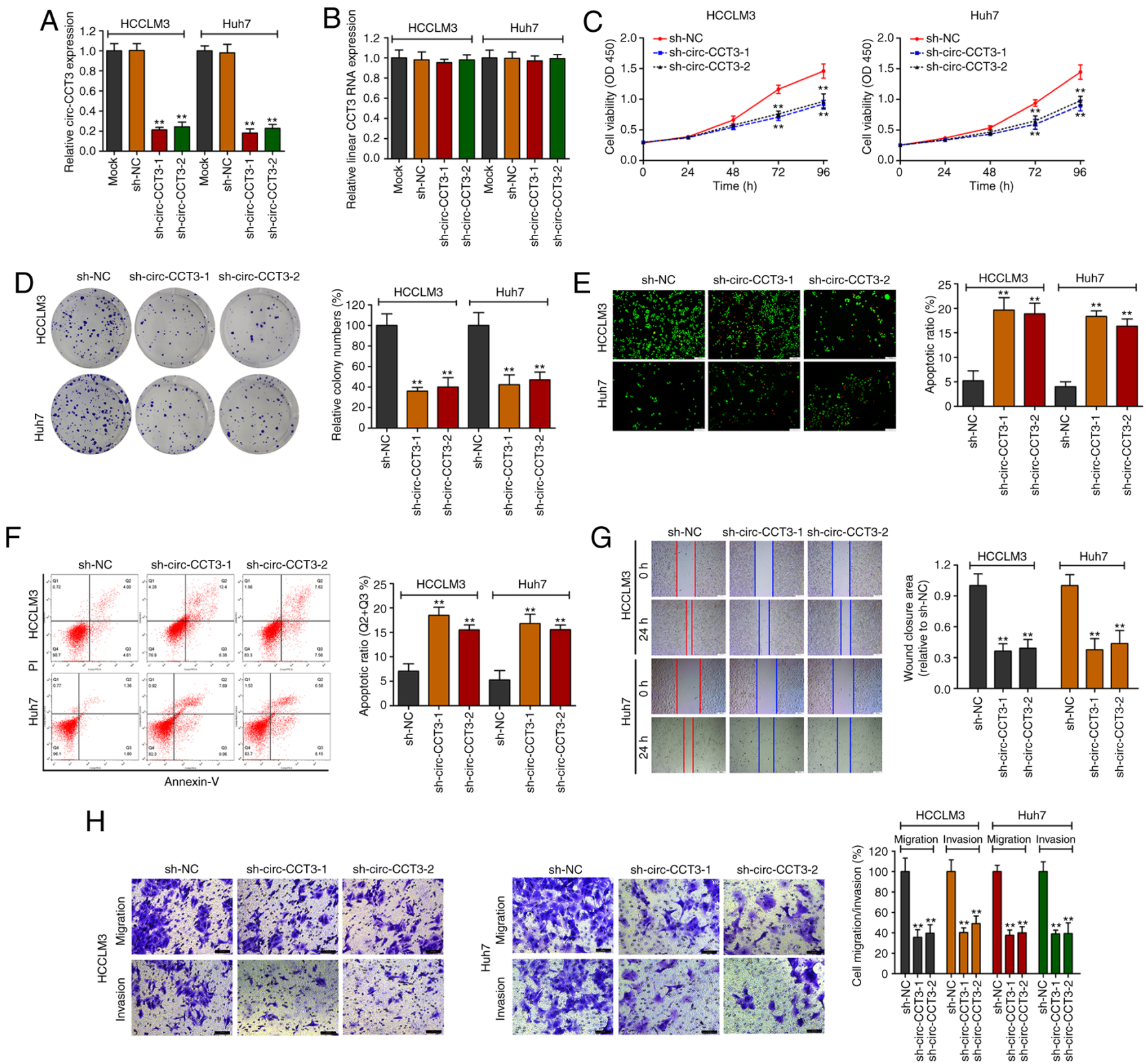

Figure 2. circ-CCT3 contributes to HCC cell progression. (A) Relative expression of circ-CCT3 was assessed by RT-qPCR following transfection in HCCLM3 and Huh7 cells. (B) Relative expression of CCT3 mRNA was assessed by RT-qPCR following transfection in HCCLM3 and Huh7 cells. (C) Cell viability was assessed after silencing circ-CCT3 in HCCLM3 and Huh7 cells by CCK-8. ${ }^{* *} \mathrm{P}<0.01$ vs. sh-NC. (D) Clone-forming ability was assessed after silencing circ-CCT3 in HCCLM3 and Huh7 cells by a colony formation assay. Cell apoptosis was detected after silencing of circ-CCT3 in HCCLM3 and Huh7 cells by (E) AO/EB staining (scale bar, $100 \mu \mathrm{m}$ ) and (F) flow cytometric assays. (G) Cell migration was assessed after silencing circ-CCT3 in HCCLM3 and Huh7 cells by a wound-healing assay (scale bar, $200 \mu \mathrm{m})(\mathrm{H})$ Cell migration and invasion were assessed after silencing circ-CCT3 in HCCLM3 and Huh7 cells by Transwell assay (scale bar, $50 \mu \mathrm{m}$ ) ${ }^{* *} \mathrm{P}<0.01$ vs. sh-NC. circ, circular; HCC, hepatocellular carcinoma; RT-qPCR, reverse transcription-quantitative PCR; $\mathrm{OD}$, optical density; $\mathrm{AO} / \mathrm{BE}$, acridine orange/ethidium bromide.

compared with the negative control (Fig. 3D). The potential target gene of miR-1287-5p was next investigated using the starBase v2.0 database. TEAD1 expression was negatively regulated by miR-1287-5p (Figs. 3E and S1A). Additionally, silencing circ-CCT3 attenuated TEAD1 expression in both HCC cell lines (Fig. 3F). TEADl mRNA was elevated in HCC samples relative to nontumorous tissues (Fig. 3G). Pearson's correlation analysis indicated a negative correlation between miR-1287-5p and TEAD1 mRNA expression (Fig. 3H). A positive correlation of circ-CCT3 and TEAD1 mRNA expression was identified in 25 pairs of HCC tissues (Fig. 3I).
Notably, TEAD1 was overexpressed in HCC cells compared with normal cells (Fig. 3J). There are three potential binding sites for miR-1287-5p within the 3'-UTR of TEAD1 (Fig. 3K). To validate the binding between the TEAD1 3'-UTR and miR-1287-5p, constructs for wt-TEAD1 3'-UTR (Luc wt) and mut-TEAD1 3'-UTR (Luc mut; Fig. 3K-L) were generated. miR-1287-5p overexpression reduced luciferase activity in cells with wt-TEAD1 3'-UTR compared to cells with mutated binding sites for miR-1287-5p (Luc mut2/mut3). Furthermore, simultaneously mutating sites 2 and 3 did not affect luciferase activity. The above results indicated that miR-1287-5p could 
A

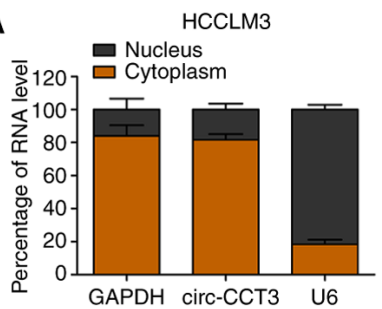

$\mathrm{D}$

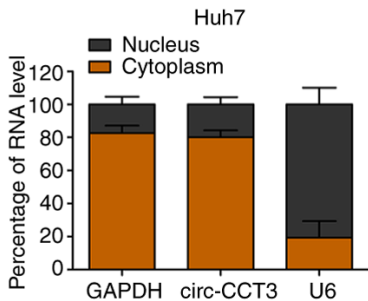

GAPDH circ-ССТ3 U6
B

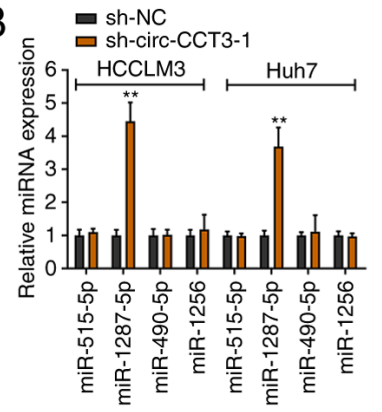

$E$ 1111111 UAGGUCGU-5 circ-CCT3 (128-134) mut 5'-AGAUUCAAGUCCAGCAAGGUCCG-3'

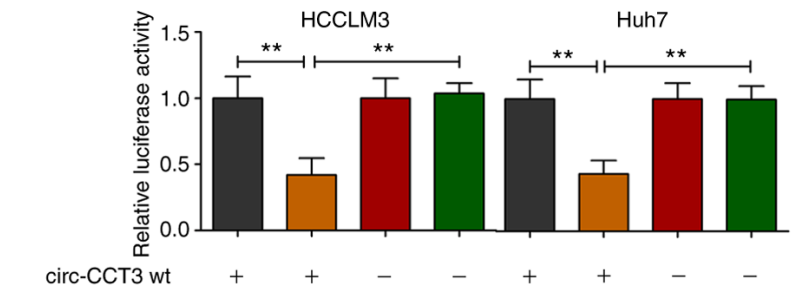

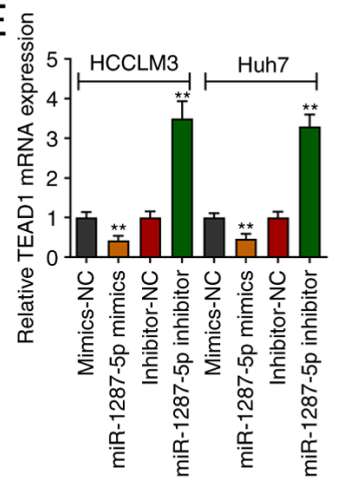

$\mathrm{F}$ 흥

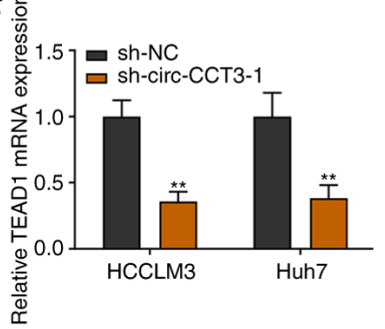

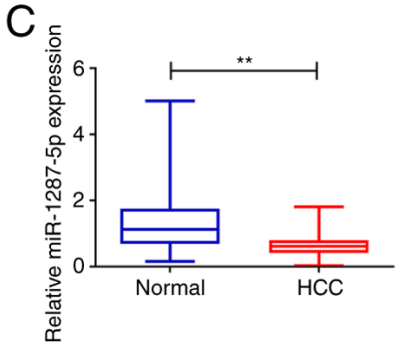

circ-ССТ3 mut $\quad-\quad-\quad+\quad+\quad-\quad-\quad+\quad+$

Mimics-NC

G

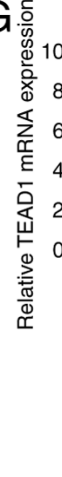

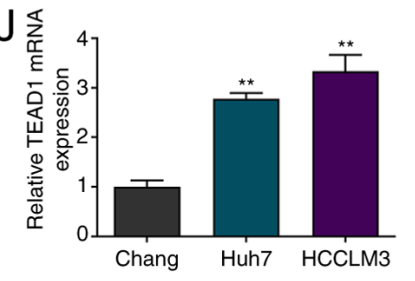

Relative circ-ССТ3 expression

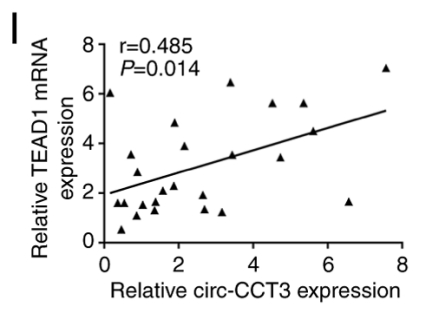

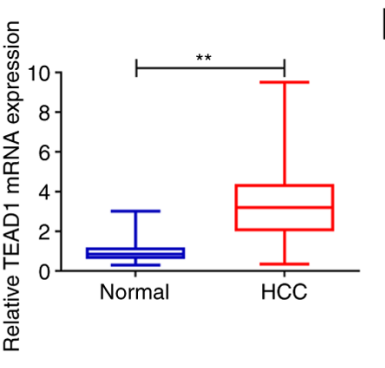

$\mathrm{L}$
$\mathrm{H}_{\text {응 }}$

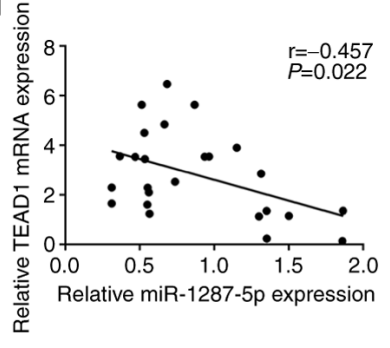

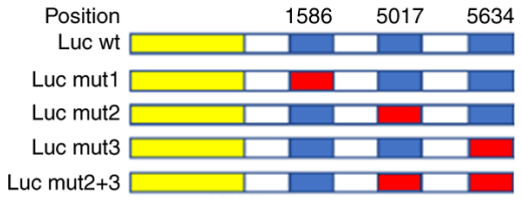

\footnotetext{
K TEAD1 3'-UTR (1586-1591) wt 5'-AACAGGGAGUGCUCCUCCAGCU-3' 111111 miR-1287-5p 3'-CUGAGCUUGGUGACUAGGUCGU-5' TEAD1 3'-UTR (1586-1591) mut 5'-AACAGGGAGUGCUCC AGGUCGU-3' TEAD1 3'-UTR (5017-5022) wt 5'-CCUUUGGUUUGAGAUUCCAGCU-3' miR-1287-5p 3'-CUGAGCUUGGUGACUAGGUCGU-5 TEAD1 3'-UTR (5017-5022) mut 5'-CCUUUGGUUUGAGAUAGGUCGU-3' TEAD1 3'-UTR (5634-5639) wt 5'-AUUUUCAAAGAAUGUUCCAGCC-3' miR-1287-5p 3'-CUGAGCUUGGUGACUAGGUCGU-5'

TEAD1 3'-UTR (5634-5639) mut 5'-AUUUUCAAAGAAUGU $\widehat{A G G U C G C} C-3^{\prime}$
}
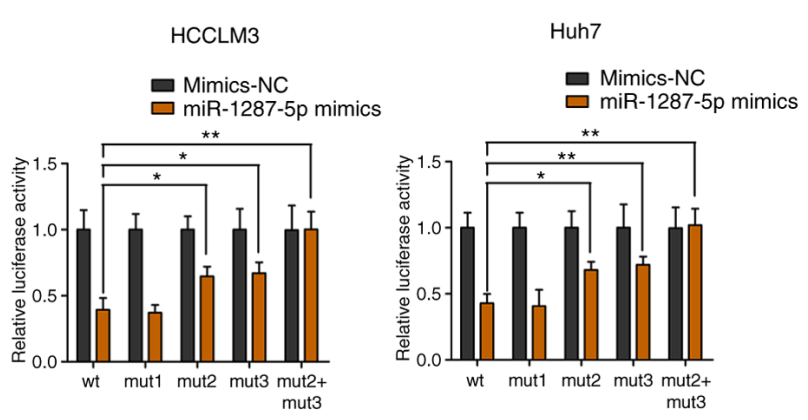

Figure 3. circ-CCT3 sponges miR-1287-5p to regulate TEAD1 expression. (A) RT-qPCR detection of the percentage of circ-CCT3 in the cytoplasmic and nuclear fractions of HCCLM3 and Huh7 cells. (B) Relative miRNA expression was assessed after silencing circ-CCT3 in HCCLM3 and Huh7 cells by RT-qPCR. ${ }^{* *} \mathrm{P}<0.01$ vs. sh-NC. (C) Relative expression of miR-1287-5p in HCC/nontumorous tissues. (D) The interaction between circ-CCT3 and miR-1287-5p was assessed in HCCLM3 and Huh7 cells by a dual-luciferase reporter assay. (E) Relative TEAD1 mRNA expression was assessed following transfection in HCCLM3 and Huh7 cells by RT-qPCR. ${ }^{* *} \mathrm{P}<0.01$ vs. mimics-NC/inhibitor-NC. (F) Relative TEAD1 mRNA expression was assessed after silencing circ-CCT3 in HCCLM3 and Huh7 cells by RT-qPCR. (G) Relative expression of TEAD1 mRNA in HCC/nontumorous tissues. Correlation analysis of TEAD1 mRNA, (H) circ-CCT3 and (I) miR-1287-5p expression in HCC/nontumorous tissues. (J) Relative expression of TEAD1 mRNA in HCC/nontumorous tissues by RT-qPCR. (K) Schematic illustration showing the TEAD1 3'-UTR of luciferase reporters and (L) reporter assays showing the luciferase activity of luc-wt/luc mut1-3 in HCCLM3 and Huh7 cells. The blue bar indicates the wild-type site without mutation. The red bar indicates the mutated site. The yellow bar indicates the vector. ${ }^{*} \mathrm{P}<0.05,{ }^{* * *} \mathrm{P}<0.01$. circ, circular; miR, microRNA; TEAD1, TEA domain transcription factor 1; RT-qPCR, reverse transcription-quantitative PCR; HCC, hepatocellular carcinoma; wt, wild-type; mut, mutated. 

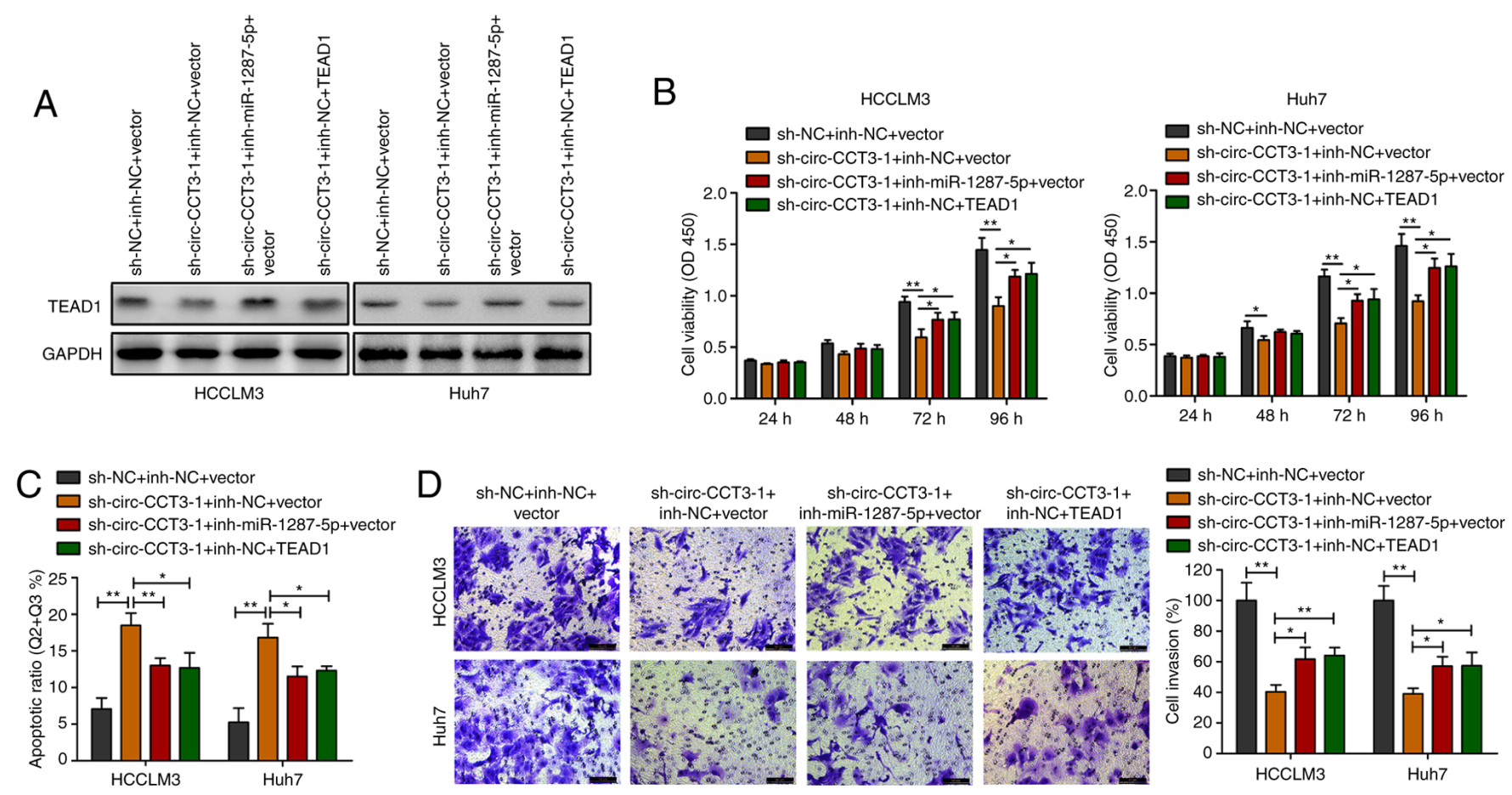

Figure 4. circ-CCT3 promotes HCC cell progression by targeting the miR-1287-5p/TEAD1 axis. (A) The protein level of TEAD1 was assessed by western blotting following transfection in HCCLM3 and Huh7 cells. (B) A CCK-8 assay was conducted to evaluate the viability of HCCLM3 and Huh7 cells following transfection. (C) A flow cytometric assay was conducted to evaluate the apoptosis of HCCLM3 and Huh7 cells following transfection. (D) Transwell assays were conducted to evaluate the invasion of HCCLM3 and Huh7 cells following transfection (scale bar, $50 \mu \mathrm{m}$ ). ${ }^{*} \mathrm{P}<0.05,{ }^{* * *} \mathrm{P}<0.01$. circ, circular; HCC, hepatocellular carcinoma; miR, microRNA; TEAD1, TEA domain transcription factor 1; sh, short hairpin; NC, negative control; inh, inhibitor.

interact with the second (5017-5022) and third (5634-5639) predicted sites of the 3'-UTR of TEAD1 (Fig. 3L).

circ-CCT3 executes oncogenic properties by targeting the miR-1287-5p/TEAD1 axis. sh-TEAD1 and TEAD1 vectors were used to silence/overexpress TEAD1 expression in HCC cells (Fig. S1B). HCC cells were co-transfected with sh-circ-CCT3-1 and miR-1287-5p inhibitor or TEAD1 vector, followed by western blotting. circ-CCT3 inhibition downregulated TEAD1 expression, whereas cotransfection with miR-1287-5p inhibitor or TEAD1 vector increased TEAD1 expression levels (Fig. 4A). CCK-8 and Transwell experiments showed that decreasing miR-1287-5p or elevating TEAD1 reversed the inhibition of HCC cell viability and invasion caused by sh-circ-CCT3-1 (Fig. 4B and D). Flow cytometric analysis demonstrated that knockdown of circ-CCT3 triggered HCC cell apoptosis. However, this effect was partially reversed by silencing miR-1287-5p or upregulating TEAD1 (Fig. 4C).

TEADI activates PTCH1 and LOX transcription in HCC. RNA-seq was performed to explore the potential target gene of TEAD1 in HCCLM3 cells (Fig. 5A). TEAD1 was ectopically expressed by transfection with the TEAD1 vector (Fig. 5B). PTCH1 and LOX expression levels were significantly elevated upon TEAD1 overexpression (Fig. 5B). Silencing of TEAD1 led to PTCH1 and LOX depletion (Fig. 5C). In addition, TEAD1 expression was positively correlated with PTCH1 and LOX expression levels analyzed with the TCGA dataset (Fig. 5D). Moreover, PTCH1 and LOX expression levels were markedly elevated in HCC tissues compared with nontumorous tissues analyzed with the TCGA dataset (Fig. 5E).
As shown in Fig. 5F, TEAD1 was predicted to bind to the promoter regions of PTCH1 and LOX. ChIP revealed significant TEAD1-binding activity on the endogenous PTCH1/LOX promoter region (Fig. 5G). A luciferase reporter assay demonstrated that TEAD1 could bind to the predicted binding sites of the PTCH1 and LOX promoters (Fig. 5H and I).

\section{Discussion}

In the present study, a circRNA microarray analysis was conducted to explore the dysregulated circRNAs in HCC tissues. circ-CCT3 was identified as the most upregulated circRNA by RT-qPCR. Therefore, the present study focused on a novel identified circRNA, circ-CCT3, originating from chr1 (156303337-156304709) of the host gene CCT3. circRNAs possess the modulatory potency of target genes (16) and act as promising biomarkers; therefore, a number of circRNAs have been explored in the onset and progression of diverse types of cancer $(17,18)$. Several circRNAs may be used as potential diagnostic or prognostic biomarkers in human cancers (18). The present study also identified circ-CCT3 as an unfavorable prognostic marker for patients with HCC.

The functions of circ-CCT3 in HCC cell progression were assessed. A knockdown study indicated that depleted circ-CCT3 inhibited cell proliferation, migration and invasion and triggered cell apoptosis. Previous studies revealed that miR-1287-5p serves an important role in breast (19), colorectal (20) and cervical (21) cancers. A previous study indicated that miR-1287-5p inhibits HCC progression by targeting PIK3R3 (22). The present study found that knockdown of circ-CCT3 enhanced miR-1287-5p expression and 
A

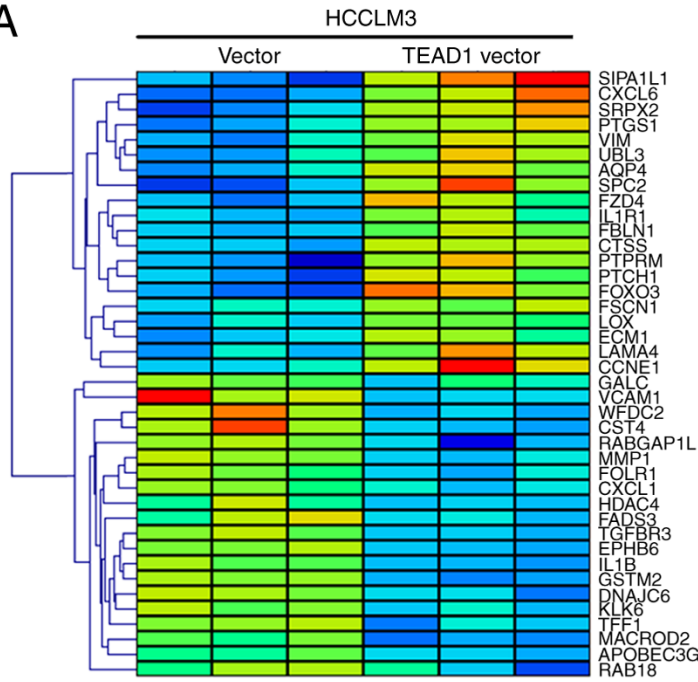

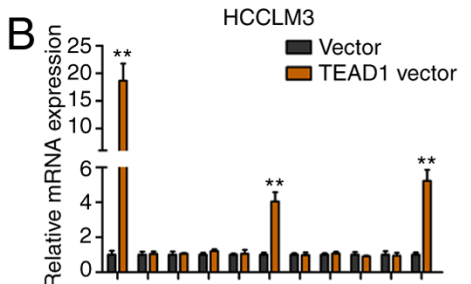

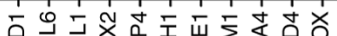
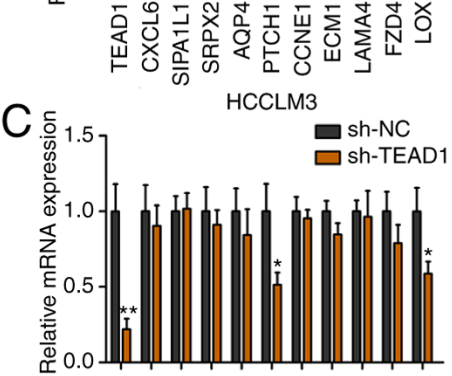

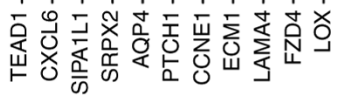

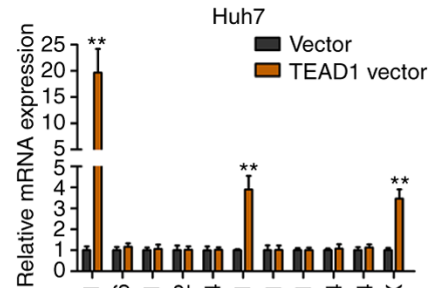

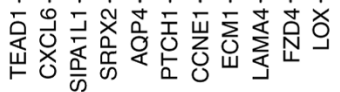
Huh7

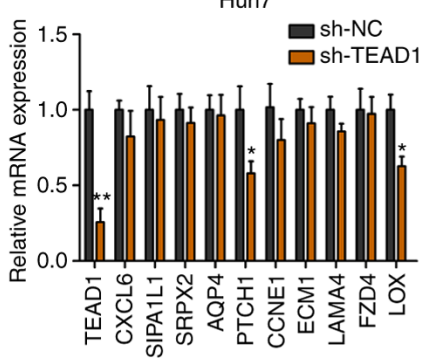

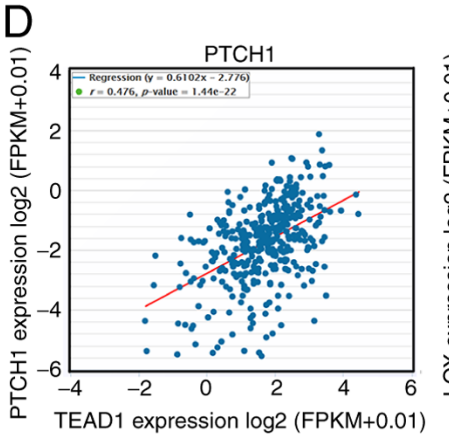

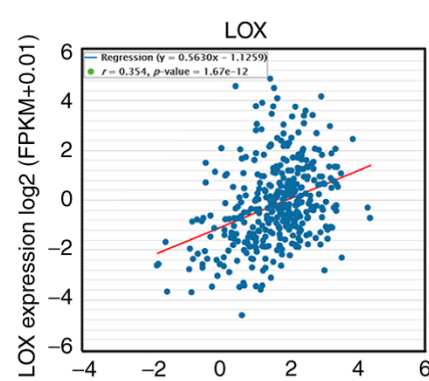

TEAD1 expression log2 (FPKM+0.01)
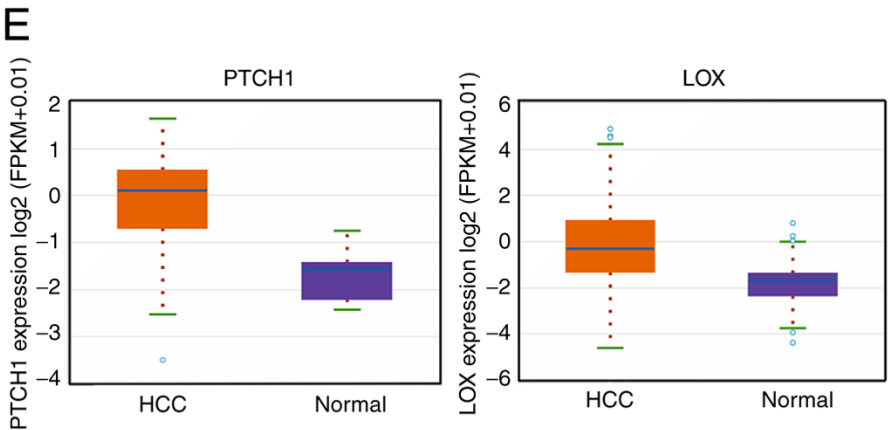

FPTCH1 promoter
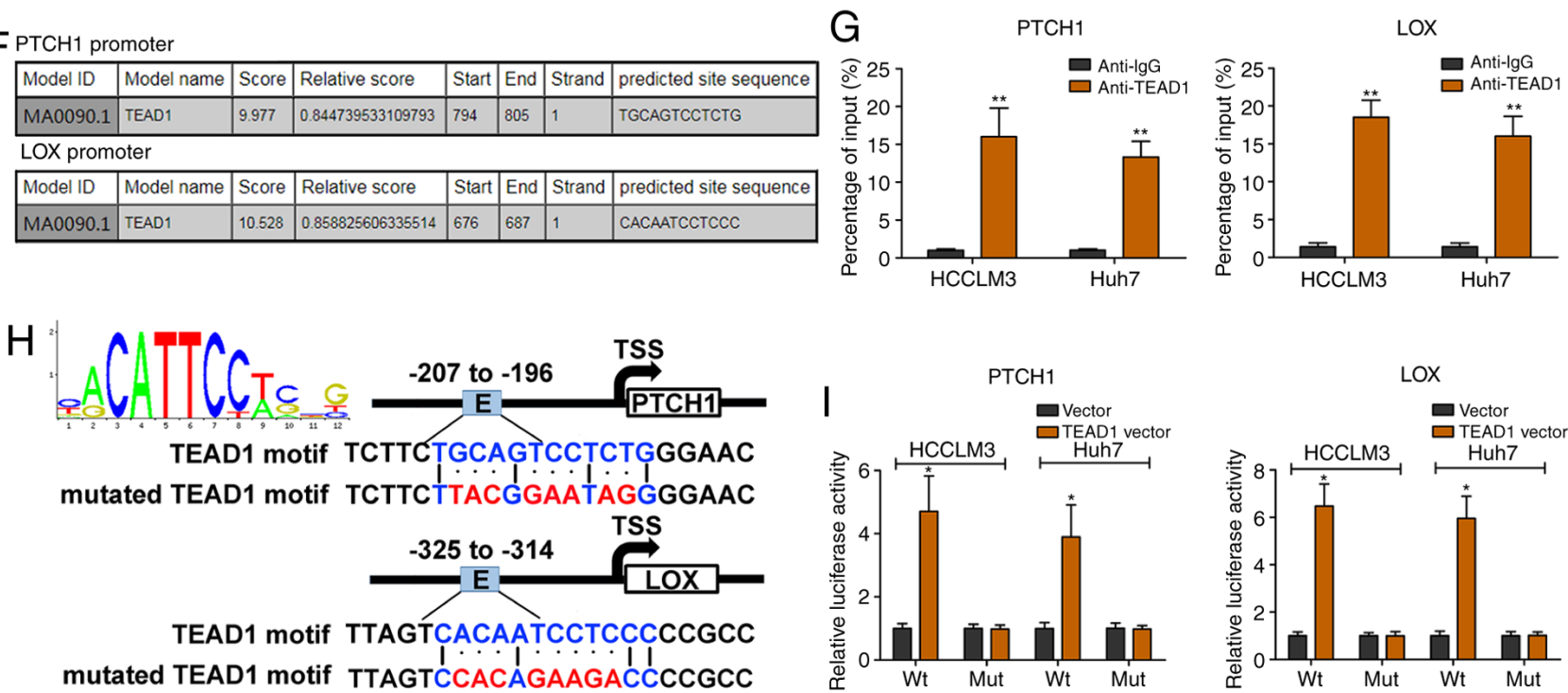

Figure 5. TEAD1 activates PTCH1 and LOX transcription. (A) Clustered heatmap showing the top 20 up/downregulated mRNA upon TEAD1 overexpression. (B and C) Relative target gene expression was assessed after overexpression/silencing of TEAD1 expression in HCCLM3 and Huh7 cells. (D) Correlations of TEAD1 and PTCH1/LOX expression analyzed with the TCGA dataset. (E) PTCH1/LOX expression in HCC/nontumorous tissues analyzed with the TCGA data. (F) TEAD1 binding site prediction in the promoter regions of PTCH1 and LOX. (G) ChIP-RT-qPCR analysis of TEAD1 occupancy in the PTCH1 and LOX promoters. (H and I) The binding ability between TEAD1 and the PTCH1/LOX promoter was measured by a luciferase reporter assay in HCC cells. ${ }^{*} \mathrm{P}<0.05,{ }^{* *} \mathrm{P}<0.01$. TEAD1, TEA domain transcription factor 1; PTCH1, patched 1; LOX, lysyl oxidase; TCGA, The Cancer Genome Atlas; ChIP, chromatin immunoprecipitation; RT-qPCR, reverse transcription-quantitative PCR.

that downregulation of miR-1287-5p resulted in a reversal effect of sh-circ-CCT3-1 in HCC cells.

It appeared that the 3'-UTR of TEAD1 contained the binding sites for miR-1287-5p, which was verified by a dual-luciferase reporter gene assay. In most cases, miRNAs regulate mRNA expression at the post-transcriptional level. circRNAs can act as sponges of miRNAs, thus indirectly regulating downstream mRNAs (23). TEAD1 transcriptional activity is 
widely believed to be modulated by the presence or absence of nuclear YAP/TAZ (24). Nevertheless, several studies have shown that TEAD itself is regulated through other mechanisms (25). It has been firmly established that TEAD1 acts as a pleiotropic transcription factor to control cell proliferation, apoptosis, metabolism, adhesion, DNA replication, differentiation and angiogenesis $(26,27)$. The present study observed that circ-CCT3 elevated TEAD1 expression by sponging miR-1287-5p in HCC. Functional experiments showed that the inhibitory effect of sh-circ-CCT3-1 on cell proliferation and invasion was attenuated by co-transfection with the TEAD1 vector, indicating the circ-CCT3/miR-1287-5p/TEAD1 regulatory axis in HCC. The TEAD1 oncogene is broadly overexpressed in a number of late-stage cancers and is often associated with tumorigenesis by causing inappropriate gene expression (28). The results of the present study suggested that up/downregulation of TEAD1 resulted in a significant increase/decrease in PTCH1 and LOX expression. PTCH1 is involved in cancer progression and chemoresistance (29). PTCH1 is the Hedgehog $(\mathrm{HH})$ receptor at the cell surface or in primary cilia, which binds to $\mathrm{HH}$ to initiate ligand-dependent signaling (30). Several studies have indicated its important role in the generation and transduction of $\mathrm{HH}$ signaling $(31,32)$. LOX is one of five members of the LOX family. It serves a primary, catalytic activity-related, role in the assembly of the extracellular matrix, a dynamic structural and regulatory framework that is essential for cell fate, differentiation and communication $(33,34)$. ChIP and luciferase reporter assays suggested that TEAD1 could directly bind to the promoter regions of PTCH1 and LOX, thereby elevating their transcription levels.

In summary, circ-CCT3 acted as a sponge for miR-1287-5p to enhance TEAD1 expression, which subsequently contributed to the activation of PTCH1 and LOX and consequently promotes tumorigenesis and progression. The present study identified a novel circRNA, circ-CCT3, which may be used as a potential therapeutic target for HCC.

\section{Acknowledgements}

Not applicable.

\section{Funding}

The present study was supported by the Research Foundation of Qiqihar Academy of Medical Sciences (grant no. QMSI2019M-27).

\section{Availability of data and materials}

The datasets used in the present study are available from the corresponding author.

\section{Authors' contributions}

WL, TZ, GD, LH, BZ, JY, YP, FG, LZ and MZ performed the experiments. WL, MZ, QY, HL, YW and CZ analyzed and interpreted the data. WL, YW and CZ wrote the manuscript. WL and TZ confirm the authenticity of all the raw data. All authors read and approved the final manuscript.

\section{Ethics approval and consent to participate}

The present study was authorized by the Institutional Review Board of The Second Affiliated Hospital of Qiqihar Medical University and written informed consent was obtained from all patients.

\section{Patient consent for publication}

Not applicable.

\section{Competing interests}

The authors declare that they have no competing interests.

\section{References}

1. Bray F, Ferlay J, Soerjomataram I, Siegel RL, Torre LA and Jemal A: Global cancer statistics 2018: GLOBOCAN estimates of incidence and mortality worldwide for 36 cancers in 185 countries. CA Cancer J Clin 68: 394-424, 2018.

2. Kalogeridi MA, Zygogianni A, Kyrgias G, Kouvaris J, Chatziioannou S, Kelekis N and Kouloulias V: Role of radiotherapy in the management of hepatocellular carcinoma: A systematic review. World J Hepatol 7: 101-112, 2015.

3. Khemlina G, Ikeda S and Kurzrock R: The biology of Hepatocellular carcinoma: Implications for genomic and immune therapies. Mol Cancer 16: 149, 2017.

4. Bartha I, di Iulio J, Venter JC and Telenti A: Human gene essentiality. Nat Rev Genet 19: 51-62, 2018.

5. Xiong DD, Dang YW, Lin P, Wen DY, He RQ, Luo DZ, Feng ZB and Chen G: A circRNA-miRNA-mRNA network identification for exploring underlying pathogenesis and therapy strategy of hepatocellular carcinoma. J Transl Med 16: 220, 2018.

6. Zhang B, Liu Z, Cao K, Shan W, Liu J, Wen Q and Wang R: circ-SPECC1 modulates TGF 32 and autophagy under oxidative stress by sponging miR-33a to promote hepatocellular carcinoma tumorigenesis. Cancer Med 9: 5999-6008, 2020.

7. Afify AY, Ibrahim SA, Aldamsisi MH, Zaghloul MS, El-Ekiaby N and Abdelaziz AI: Competing endogenous RNAs in hepatocellular carcinoma-the pinnacle of rivalry. Semin Liver Dis 39: 463-475, 2019.

8. Wilczynska A and Bushell M: The complexity of miRNA-mediated repression. Cell Death Differ 22: 22-33, 2015.

9. Bitarte N, Bandres E, Boni V, Zarate R, Rodriguez J, Gonzalez-Huarriz M, Lopez I, Javier Sola J, Alonso MM, Fortes P, et al: MicroRNA-451 is involved in the self-renewal, tumorigenicity, and chemoresistance of colorectal cancer stem cells. Stem Cells 29: 1661-1671, 2011.

10. Heng BC, Zhang X, Aubel D, Bai Y, Li X, Wei Y, Fussenegger M and Deng $X$ : An overview of signaling pathways regulating YAP/TAZ activity. Cell Mol Life Sci 78: 497-512, 2021.

11. R Core Team: (v3.14.0; 2016): R: A language and environment for statistical computing. R Foundation for Statistical Computing, Vienna, Austria. https://www.gbif.org/tool/81287/r-a-language-and-environm ent-for-statistical-computing. Accessed February 10, 2015.

12. Livak KJ and Schmittgen TD: Analysis of relative gene expression data using real-time quantitative PCR and the 2(-Delta Delta C(T)) Method. Methods 25: 402-408, 2001.

13. Yu J, Zhang B, Zhang H, Qi Y, Wang Y, Wang W, Wang Y and Wang Y: E2F1-induced upregulation of long non-coding RNA LMCD1-AS1 facilitates cholangiocarcinoma cell progression by regulating miR-345-5p/COL6A3 pathway. Biochem Biophys Res Commun 512: 150-155, 2019.

14. Li JH, Liu S, Zhou H, Qu LH and Yang JH: starBase v2.0: Decoding miRNA-ceRNA, miRNA-ncRNA and protein-RNA interaction networks from large-scale CLIP-Seq data. Nucleic Acids Res 42D: D92-D97, 2014.

15. Dudekula DB, Panda AC, Grammatikakis I, De S, Abdelmohsen K and Gorospe M: CircInteractome: A web tool for exploring circular RNAs and their interacting proteins and microRNAs. RNA Biol 13: 34-42, 2016. 
16. Memczak S, Jens M, Elefsinioti A, Torti F, Krueger J, Rybak A Maier L, Mackowiak SD, Gregersen LH, Munschauer M, et al: Circular RNAs are a large class of animal RNAs with regulatory potency. Nature 495: 333-338, 2013.

17. Xu Y, Yao Y, Zhong X, Leng K, Qin W, Qu L, Cui Y and Jiang X: Downregulated circular RNA hsa_circ_0001649 regulates proliferation, migration and invasion in cholangiocarcinoma cells. Biochem Biophys Res Commun 496: 455-461, 2018.

18. Han D, Li J, Wang H, Su X, Hou J, Gu Y, Qian C, Lin Y, Liu X, Huang M, et al: Circular RNA circMTO1 acts as the sponge of microRNA-9 to suppress hepatocellular carcinoma progression. Hepatology 66: 1151-1164, 2017.

19. Schwarzenbacher D, Klec C, Pasculli B, Cerk S, Rinner B, Karbiener M,Ivan C,Barbano R,Ling H,Wulf-Goldenberg A, et al: miR-1287-5p inhibits triple negative breast cancer growth by interaction with phosphoinositide 3-kinase $\mathrm{CB}$, thereby sensitizing cells for PI3Kinase inhibitors. Breast Cancer Res 21: 20, 2019.

20. Cui G, Zhao H and Li L: Long noncoding RNA PRKCQ-AS1 promotes CRC cell proliferation and migration via modulating miR-1287-5p/YBX1 axis. J Cell Biochem 121: 4166-4175, 2020.

21. Ji F, Du R, Chen T, Zhang M, Zhu Y, Luo X and Ding Y: Circular RNA circSLC26A4 Accelerates Cervical Cancer Progression via miR-1287-5p/HOXA7 Axis. Mol Ther Nucleic Acids 19: 413-420, 2020

22. Lu J, Tang L, Xu Y, Ge K, Huang J, Gu M, Zhong J and Huang Q: mir-1287 suppresses the proliferation, invasion, and migration in hepatocellular carcinoma by targeting PIK3R3. J Cell Biochem 119: 9229-9238, 2018.

23. Han TS, Hur K, Cho HS and Ban HS: Epigenetic Associations between lncRNA/circRNA and miRNA in Hepatocellular Carcinoma. Cancers (Basel) 12: 2622, 2020.

24. Mammoto A, Muyleart M, Kadlec A, Gutterman D and Mammoto T: YAP1-TEAD1 signaling controls angiogenesis and mitochondrial biogenesis through PGC1 $\alpha$. Microvasc Res 119: 73-83, 2018.

25. Landin-Malt A, Benhaddou A,Zider A and Flagiello D: An evolutionary, structural and functional overview of the mammalian TEAD1 and TEAD2 transcription factors. Gene 591: 292-303, 2016.
26. Zhou Y, Huang T, Zhang J, Wong CC, Zhang B, Dong Y, Wu F, Tong JHM, Wu WKK, Cheng ASL, et al: TEAD1/4 exerts oncogenic role and is negatively regulated by miR-4269 in gastric tumorigenesis. Oncogene 36: 6518-6530, 2017.

27. Tome-Garcia J, Erfani P, Nudelman G, Tsankov AM, Katsyv I, Tejero R, Bin Zhang, Walsh M, Friedel RH, Zaslavsky E, et al: Analysis of chromatin accessibility uncovers TEAD1 as a regulator of migration in human glioblastoma. Nat Commun 9: 4020, 2018

28. Fan Y, Gao Y, Rao J, Wang K, Zhang F and Zhang C: YAP-1 promotes tregs differentiation in hepatocellular carcinoma by enhancing TGFBR2 transcription. Cell Physiol Biochem 41: 1189-1198, 2017.

29. Olesen UH, Bojesen S, Gehl J and Haedersdal M: Anticancer drugs and the regulation of Hedgehog genes GLI1 and PTCH1, a comparative study in nonmelanoma skin cancer cell lines. Anticancer Drugs 28: 1106-1117, 2017.

30. Qi C, Di Minin G, Vercellino I, Wutz A and Korkhov VM: Structural basis of sterol recognition by human hedgehog receptor PTCH1. Sci Adv 5: eaaw6490, 2019.

31. Skoda AM, Simovic D, Karin V, Kardum V, Vranic S and Serman L: The role of the Hedgehog signaling pathway in cancer: A comprehensive review. Bosn J Basic Med Sci 18: 8-20, 2018.

32. Qi X and Li X: Mechanistic insights into the generation and transduction of hedgehog signaling. Trends Biochem Sci 45: 397-410, 2020

33. Laczko R and Csiszar K: Lysyl Oxidase (LOX): Functional contributions to signaling pathways. Biomolecules 10: 1093, 2020.

34. Saatci O, Kaymak A, Raza U, Ersan PG, Akbulut O, Banister CE, Sikirzhytski V, Tokat UM, Aykut G, Ansari SA, et al: Targeting lysyl oxidase (LOX) overcomes chemotherapy resistance in triple negative breast cancer. Nat Commun 11: 2416, 2020 